Para enlazar con este artículo / To link to this article:

http://dx.doi.org/10.6035/MonTI.2019.ne4.12

Para citar este artículo / To cite this article:

PARRA LÓPEZ, Guillermo \& Eduard Bartoll Teixidor. (2019) "El tesoro lingüístico de Gollum. El uso del idiolecto en la caracterización de la identidad de los personajes de ficción y su traducción para el doblaje." En: Pérez L. DE Heredia, María \& Irene Higes Andino (eds.) 2019. Multilingüismo y representación de las identidades en textos audiovisuales / Multilingualism and representation of identities in audiovisual texts. MonTI Special Issue 4, pp. 343-370.

\title{
EL TESORO LINGÜÍSTICO DE GOLLUM. EL USO DEL IDIOLECTO EN LA CARACTERIZACIÓN DE LA IDENTIDAD DE LOS PERSONAJES DE FICCIÓN Y SU TRADUCCIÓN PARA EL DOBLAJE
}

\author{
Guillermo Parra López \\ guillermo.parra@upf.edu \\ Universitat Pompeu Fabra, Barcelona \\ Eduard Bartoll Teixidor \\ eduard.bartoll@upf.edu \\ Universitat Pompeu Fabra, Barcelona
}

\section{Resumen}

El objetivo del presente artículo es reflexionar sobre la naturaleza del idiolecto y su tratamiento en la traducción mediante el lenguaje de Gollum, uno de los personajes más reconocibles de El señor de los anillos. Tradicionalmente, el idiolecto ha recibido poca atención tanto desde el punto de vista lingüístico como del traductológico, quizás debido a su esencia individual y asistemática. Sin embargo, es importante conocer las implicaciones de este fenómeno para la traducción, puesto que es un elemento fundamental en la configuración de la identidad de los personajes en las obras de ficción. En este estudio analizamos detenidamente una selección de muestras del idiolecto de Gollum obtenidas de la adaptación cinematográfica de la Trilogía y observamos el tratamiento que han recibido sus idiosincrasias lingüísticas en la versión doblada al español.

\begin{abstract}
"Gollum's linguistic precious. The use of idiolect in the depiction of the identity of fictional characters and its translation for dubbing"

The aim of this article is to reflect on the nature of the idiolect and its treatment in translation through the language of Gollum, one of the most notable characters in The Lord of the Rings. Idiolects have historically received little attention from both linguistics and translation studies, probably because of their individual and asystematic essence.
\end{abstract}


Nevertheless, it is important to know the implications of this phenomenon for translation, since it is a fundamental element in the depiction of characters' identity in fiction works. This study is devoted to analyze a selection of samples from Gollum's idiolect obtained from the film adaptation of the Trilogy and to observe how his linguistic idiosyncrasies have been dealt with in the Spanish dubbed version.

Palabras clave: Idiolecto. Traducción. Doblaje. Gollum. Variación lingüística.

Keywords: Idiolect. Translation. Dubbing. Gollum. Language variation. 


\section{Introducción}

Al contrario que otras formas de variación lingüística como el geolecto o el sociolecto, analizados en lingüística y traducción, el idiolecto ha recibido poca atención por parte de ambas disciplinas, probablemente por su esencia individual y asistemática. Siguiendo la distinción de Corrius \& Zabalbeascoa (2011), creemos que el idiolecto puede considerarse como una tercera lengua (L3), distinta de la lengua origen ( $\mathrm{LO}$ o L1) y la lengua meta (LM o L2). Consideramos que su estudio es relevante para la traducción, porque es un elemento fundamental para identificar a los personajes en obras de ficción. El desconocimiento de sus particularidades conlleva que se trate de forma equivocada.

Este artículo pretende profundizar en la naturaleza de esta forma de variación lingüística y en su tratamiento en la traducción audiovisual. Nos centramos en uno de los ejemplos de idiolecto por excelencia: el habla de Gollum, personaje de la trilogía de El señor de los anillos conocido por su forma de expresarse. Analizaremos la versión extendida de las dos últimas películas de la serie (The Lord of the Rings: The Two Towers, Peter Jackson 2002, y The Lord of the Rings: The Return of the King, Peter Jackson 2003), con casi todas las intervenciones del personaje, y observaremos sus rasgos idiolectales característicos y estudiaremos cómo se han trasladado al español en sus versiones dobladas (El señor de los anillos: Las dos torres y El señor de los anillos: El retorno del rey). Dadas la limitación de extensión y la tendencia habitual de los subtítulos hacia la neutralización de variedades y registros (cf. Rosa 2001), solo analizaremos las versiones dobladas. Sin ser estas soluciones necesariamente extrapolables a otras obras y modalidades de traducción, creemos que estudiar un caso tan marcado como el de Gollum contribuye a la reflexión sobre este fenómeno.

La metodología seguida consta de cinco fases: 1) una breve definición de la traducción audiovisual y, en especial, de la modalidad del doblaje; 2) un repaso bibliográfico sobre el concepto de idiolecto y sus implicaciones para la traducción; 3) la recopilación de muestras del idiolecto de Gollum a partir de la versión original de las películas; 4) el análisis de las idiosincrasias lingüísticas de Gollum y de sus interrelaciones, y 5) la comparación con la traducción al español para doblaje de dichas idiosincrasias. 


\section{Traducción audiovisual: el doblaje}

Según Bartoll (2015: 41), la traducción audiovisual es la translación de textos audiovisuales, que son los que transmiten la información de manera dinámicotemporal mediante el canal acústico, el canal visual o ambos a la vez. Los textos audiovisuales se presentan a través de distintos géneros, como la ficción, los documentales, la publicidad, etc. Aunque en este estudio solo se tratará el doblaje, la traducción audiovisual se lleva a cabo mediante diversas modalidades como la subtitulación, las voces superpuestas, la interpretación simultánea, la interpretación consecutiva, el remake, la intertitulación, la audiodescripción, el resumen escrito, la narración o el comentario (Bartoll 2015: 63-65).

Como la información en estos textos se transmite por ese doble canal, acústico y visual, y muchas veces los productos audiovisuales de ficción pretenden reflejar la realidad, la lengua ofrece muchos elementos característicos de la oralidad. Aunque se trate de una oralidad prefabricada o fingida (Chaume 2001; Baños-Piñero y Chaume 2009), se busca resultar creíble si la situación también pretende serlo. A pesar de ser un texto no espontáneo y elaborado por guionistas, presenta elementos de oralidad como construcciones coloquiales, juegos de palabras, pronunciación relajada y expresiones figuradas.

Toda traducción audiovisual debe aspirar a mantener, en la medida de lo posible, el grado de verosimilitud del texto origen (TO). Es necesario que el traductor detecte los elementos propios del supuesto discurso oral y busque estrategias para transferirlos a su lengua (García de Toro 2009: 138). Además, el texto meta (TM) debe ajustarse a la imagen y evitar incoherencias o contradicciones, aunque las restricciones varían en función de la modalidad. En el caso del doblaje, Chaume (2012: 68) explica:

Synchronization is one of the features of translation for dubbing that consists of matching the target language translation and the articulatory and body movements of the screen actors and actresses, and ensuring that the utterances and pauses in the translation match those of the source text.

Chaume (2012: 68) establece tres tipos de sincronización: fonética o sincronía labial, cinética o de movimiento corporal e isocronía o sincronía entre los enunciados y las pausas. El incumplimiento de estos principios podría romper la denominada "suspensión de incredulidad" ("willing suspension of disbelief"; Coleridge \& Shawcross 1907) y causar extrañeza en el público.

El doblaje consta de tres fases: traducción del guion, ajuste del texto de la traducción a los citados movimientos e interpretación del texto ajustado por los actores (Chaume 2003: 17). Quizás no todas las personas implicadas en el proceso conozcan la LO, por lo que el traductor debe prever las dificultades y 
anticiparse a ellas, ofreciendo alternativas para el ajustador o añadiendo indicaciones sobre las particularidades de algunos personajes, etc.

\section{Idiolecto y traducción}

\subsection{La variación lingüistica}

Mayoral (1999: 13) define la variación lingüística como "la expresión de significados potencialmente similares mediante estrategias diferentes que dan lugar a segmentos textuales distintos". Catford (1965: 86) apunta que, para considerarse variantes de una misma lengua, aparte de los rasgos específicos de la variedad en cuestión (de carácter fonético, fonológico, grafológico, gramatical o léxico), a los que denomina marcadores, deben compartir otros (gramaticales, léxicos o fonológicos) que constituyan un núcleo común.

Los expertos clasifican las diversas variedades de múltiples maneras, aunque la mayoría según la distinción básica de Catford (1965: 84-85) entre variedades relacionadas con las características permanentes del hablante y aquellas transitorias relacionadas con la situación inmediata del enunciado; lo que Hatim y Mason (1991) más tarde denominaron "dimensión del usuario" ("user-related") y "dimensión del uso" ("use-related"). El primer grupo incluye el geolecto (variedad geográfica), el cronolecto (variedad temporal), el sociolecto (variedad social), el estándar y el idiolecto (variedad individual), y el segundo recoge formas como el campo, el tenor y el modo.

\subsection{El idiolecto}

La investigación sobre el idiolecto resulta escasa comparada con la de otras variedades, por ser un fenómeno individualizado (Sánchez Iglesias 2005). Varios expertos proponen diversas definiciones para este concepto. Para el desarrollo teórico de este artículo, partimos de la formulada por dicho autor. El término idiolecto (del latín idiolectus, 'habla individual') se utiliza para designar

el conjunto de usos de una lengua característico de un individuo concreto [...]. La noción de idiolecto implica que existen variaciones no solo de un país a otro, de una región a otra, de un pueblo a otro, de una clase social a otra, sino también de una persona a otra (Sánchez Iglesias 2001: 704).

El idiolecto es "la resultante indisoluble entre una percepción particular del mundo y la forma lingüística que contiene dicha percepción" (García López 2004: 57). El valor connotativo que el hablante asigne a una palabra o expresión prima sobre el denotativo. Para Coulthard (2004), cada hablante nativo tiene su propia versión individual de la lengua que habla y escribe, con un vocabulario activo construido a lo largo de los años. Esto se manifiesta en los 
textos con elecciones distintivas e idiosincrásicas. Es de prever que el idiolecto también dependa de la situación comunicativa y evolucione con el tiempo, reflejando la experiencia personal y el desarrollo del hablante (Gregory \& Carroll 1978; Mayoral 1999).

Basándose en Alcaraz Varó \& Martínez Linares (1997), Sánchez Iglesias (2001) recoge los elementos lingüísticos donde se observa el idiolecto: del ámbito fonético-fonológico, los rasgos que constituyen la dinámica de la voz, así como los idiófonos (denominación utilizada para los sonidos identificables en el habla de una persona determinada); del sintáctico, las construcciones utilizadas con mayor o menor frecuencia, la estructura del texto, la distribución de la información y de la proporción y constitución de frases nominales y verbales; y, finalmente, del nivel léxico-semántico, el uso recurrente de determinadas unidades: todos aquellos hábitos lingüísticos que distinguen al individuo. Según Sánchez Iglesias (2001: 709):

El idiolecto adquiere identidad en la medida en que es una constante que el receptor puede identificar, y a la que le puede otorgar una funcionalidad, meramente la de individualizar al emisor.

El mismo autor sugiere la posibilidad de que el uso reiterado de las idiosincrasias constituya alguna forma de referencia cotextual, que también afectaría a la cohesión del texto.

Sobre la relación de la variedad individual con las demás variedades según el usuario, Hatim \& Mason (1997) no consideran que los idiolectos sean periféricos, como podría desprenderse de la literatura científica. Implícitamente, les otorgan una posición central dentro de la variación lingüística, al reconocer que presentan rasgos de los demás lectos. Esta dualidad entre lo individual y lo colectivo causa confusión, como explica Mayoral (1999: 101), que considera necesario distinguir entre dos planos:

a) la individualidad resultante de la suma de todos los rasgos situacionales del hablante, que en su conjunto pueden resultar únicos pero que individualmente no tienen por qué serlo;

b) la suma de los rasgos individuales idiosincrásicos.

Hatim \& Mason (1997) establecen una tipología de idiolectos según dos escalas complementarias: la de recurrencia (de transitorio a duradero) y la de funcionalidad (de afuncional a funcional) y opinan que los idiolectos duraderos y funcionales -el caso del de Gollum- presentan mayor interés para el traductor. Algunos autores consideran necesario distinguir a nivel teórico entre idiolecto y estilo. Para García de Toro (1994), el primero corresponde a los hábitos lingüísticos inconscientes, y el segundo está dotado de intencionalidad y hace referencia a elecciones motivadas. O'Donnell \& Todd (1980: 62) 
hablan de "dialecto" como el tipo de variedad entre idiolectos y consideran el estilo como tipo de variedad en los idiolectos. Sánchez Iglesias (2001: 708) propone que el idiolecto sea utilizado en un texto con intención estilística. Así, los elementos idiolectales constituirían "un rasgo de estilo al que el emisor adjudica una intencionalidad determinada". Son consideraciones teóricas que, a efectos prácticos, no influyen en cómo abordar estos elementos en la traducción, porque, según Mayoral (1999), no hay forma de determinar cuáles son voluntarios y cuáles no.

\subsection{Aspectos generales sobre la traducción del idiolecto}

Lo primero para traducir textos idiolectales es reconocer el idiolecto y, más importante, establecer la función que cumple en el entramado textual, de esta dependerá el enfoque del traductor (Sánchez Iglesias 2005). Para Catford (1965) solo deben conservarse las peculiaridades idiolectales del personaje si sirven para identificarlo, y la solución adoptada por el traductor debe ser un rasgo idiolectal "equivalente". Si la identidad del hablante carece de relevancia, su reproducción en la traducción es prescindible.

La función del texto también puede condicionar al traductor. Newmark (1988) propone que las idiosincrasias se mantengan en los escritos de autores importantes, especialmente si hay diálogo, donde la variación tiene un peso innegable, como sugiere Costa (2012). Sin embargo, Newmark opina que no sería necesario traducirlas en textos de función predominantemente referencial. El autor tiene una concepción extrema de los fenómenos idiolectales, que achaca a la "pobreza de estilo"1, y opta generalmente por su normalización. Esta posición se opone a la de Sánchez Iglesias (2005: 181), que opina que no traducir el idiolecto equivale a neutralizar al personaje: "Neutralizar que casi significa silenciar, porque despojamos a un personaje de su voz, de su elemento más característico".

Estas consideraciones, aplicadas a nuestro texto, donde predominan las funciones expresiva y poética, y el habla de Gollum constituye un medio importante de caracterización, apuntan a que sí debería tenerse en cuenta su idiolecto.

López Rúa (1997: 158) coincide con Sánchez Iglesias en que la neutralización descolora la traducción y priva al original "de parte de su intención y significado". Como alternativa a esta omisión, propone tres estrategias para traducir las estructuras lexicalizadas de los idiolectos: 1) la traducción literal, poco recomendable, porque implica una correspondencia de estructuras entre

1. "In some cases, it is not easy to distinguish between poor writing and idiolect [...] but the translator does not have to make that distinction, and merely normalises". 
las dos lenguas que raramente se da; 2) la traducción por equivalencia, recurriendo a una estructura lexicalizada en la LM con diferente forma pero mismo significado, y 3) la traducción por modificación, consistente en una paráfrasis del original, si no se encuentra un equivalente en la LM. Para García López (2004), "equivalentes" son las soluciones de la LM que logren que la traducción produzca en el polisistema meta el mismo efecto comunicativo del original.

Quizás la mayor dificultad para traducir el idiolecto se dé cuando combina características de las demás variedades (Sánchez Iglesias 2005). Este no es el caso del idiolecto de Gollum, cuya naturaleza libra al traductor de tener que situar al personaje en un contexto geográfico, social o temporal conocido, aunque sí plantea otros problemas, como el propuesto por García de Toro (1994: 95):

Conseguir que las soluciones propuestas sean homogéneas (es decir, por ejemplo, utilizando elementos reiterados), de modo que las implicaturas que se desprendan sean siempre las mismas y que el lector reconozca tales frases como pertenecientes al discurso de dicho personaje.

Al no poder mantener siempre las características idiolectales en el mismo lugar en el que aparecían en el TO, el traductor puede recurrir a la compensación en otros pasajes para atenuar la pérdida, estrategia vital en textos como el que nos ocupa. Así, puede identificarse cierta recurrencia en el TM, aunque sea en forma de "marcas idiolectales del traductor" (García de Toro 2009: 145). Sin embargo, como afirma Sánchez Iglesias (2005), la falta de homogeneidad al tratar las idiosincrasias acostumbra a ser la norma más que la excepción.

\section{Sobre Gollum y la Tierra Media}

\subsection{El autor}

John Ronald Reuel Tolkien fue un erudito británico especializado en inglés medio y antiguo. Sus escritos se caracterizan por el uso de lenguas artificiales que él mismo inventaba y por un dominio extraordinario de la lengua inglesa. Entre sus numerosas obras, destacan El Silmarillion, El hobbit y El señor de los anillos (cf. The Tolkien Society 2017).

\subsection{La Trilogía}

La trilogía de El señor de los anillos (1954-1955) está ambientada en una era prehistórica en la Tierra Media, un mundo inventado por Tolkien y habitado por multitud de razas fantásticas como elfos, enanos, hóbbits, troles y orcos, aparte de seres humanos. El señor de los anillos cuenta la odisea del hóbbit Frodo Bolsón y de sus compañeros, que deben llevar el Anillo Único, el arma más poderosa del enemigo, hasta los confines del continente para destruirlo. 
Solo así podrán librarse del mal que amenaza con apoderarse de la Tierra Media, personificado en la figura de Sauron. Si el Anillo cayera en manos equivocadas, el Señor Oscuro resurgiría.

Tanto las lenguas artificiales de Tolkien como los cronolectos del inglés desempeñan un papel diferenciador para la caracterización de la identidad de las razas ficticias de la Tierra Media. Esto demuestra la importancia de los aspectos lingüísticos en la obra, que Tolkien cuidó hasta el mínimo detalle, hasta el punto de redactar una guía con consejos e indicaciones para los traductores (cf. Tolkien 1975).

\subsection{La adaptación cinematográfica}

La adaptación cinematográfica de la Trilogía llegó entre 2001 y 2003 a cargo de Peter Jackson. La acción se dividió en tres partes, según la segmentación original de los libros: La comunidad del anillo (Peter Jackson 2001), Las dos torres y El retorno del rey. El personaje de Gollum apenas tiene relevancia en la primera película, por lo que este estudio se basa exclusivamente en las otras. En ellas, Gollum guía a Frodo, el portador del Anillo, y a Sam, su más fiel compañero, hacia Mordor, tierra del Señor Oscuro, donde deberán destruir el Anillo. Nino Matas se encargó de la traducción para el doblaje de las tres películas, en el estudio de grabación Sonoblok bajo dirección y ajuste de Miguel Ángel Jenner, según las entradas de Eldoblaje.com (2017).

\subsection{El personaje}

Después de que Sauron caiga a manos de Isildur, el rey de los hombres, y de que este muera en un asalto, el Anillo cae al fondo del río Grande. Allí permanece olvidado durante más de dos milenios hasta que los hóbbits Déagol y Sméagol lo encuentran fortuitamente mientras pescan. El Anillo se apodera enseguida de la voluntad de Sméagol, que asesina a su amigo para quedárselo. Tras este suceso, Sméagol es desterrado a la montaña, donde malvive solo durante siglos por la longevidad que le otorga el Anillo. Durante ese periodo olvida su identidad y se convierte en un ser salvaje al que los demás personajes de la Tierra Media llaman Gollum (cf. apartado 5.1). En palabras de Nagy (2006: 68-69): "El Anillo se alza como línea de demarcación entre su antigua identidad y la subjetividad empobrecida, borrada y perdida"2. Arrizabalaga (2007: 267) describe así a Gollum:

2. "The Ring stands as a demarcation line between his old identity and the impoverished, erased, lost subjectivity". 
Gollum, o Sméagol, el nombre que la criatura aún conserva de sus días previos al hallazgo del Anillo, es la versión degradada de un ser que ha quedado en el pasado. [...] Ahora padece una metamorfosis deformante que lo condena a sobrellevar una vida subterránea y permanecer oculto al resto de las criaturas de la superficie.

Gollum se debate constantemente entre el bien y el mal. Es un personaje redondo, complejo, con un papel fundamental en El señor de los anillos, "demuestra lo que le ocurre al sujeto cuando no puede ejercer ningún control sobre las fuerzas y procesos que lo determinan"3 (Nagy 2006: 59). El mismo Peter Jackson reconoce en la versión comentada de la película que, debido al valor simbólico de Gollum, la falta de solidez en su personaje habría afectado a la credibilidad de toda la serie.

\section{La traducción del idiolecto de Gollum}

Las películas de El señor de los anillos nos revelan que las peculiaridades de Gollum no se limitan a su carácter, sino que se reflejan en su idiolecto. El mundo interno del personaje se exterioriza en una forma de hablar única y característica. Nagy (2006: 59) coincide en lo siguiente:

The most remarkable thing about Gollum is definitely his language. He speaks with a general phonetic and syntactic simplicity [...]. He is often termed a 'schizophrenic' character, meaning that his language and behavior [...] are sensed to be divided and shifting.

Arrizabalaga (2007: 267) destaca su manejo defectuoso y agramatical de la lengua, que aprendió en su vida anterior en la superficie y que apenas conserva "en los monólogos de su vida ermitaña". El traductor se enfrenta al reto de reconstruir en el TM esa lengua fragmentada y olvidada, vital para caracterizar la identidad del personaje.

\subsection{El tesoro y Gollum}

Es necesario aclarar dos puntos fundamentales sobre el personaje: qué representa realmente el tesoro y qué relación tiene con el sobrenombre de Gollum. Según Nagy (2006: 60):

The word 'precious' acts as central signifier in Gollum's language. It comes up functionally integrated into sentences, but also as a sort of interjection, something which does not have any further meaning than being used in

3. "He demonstrates what happens to the subject when it cannot exert any control over the forces and processes that determine it". 
certain positions and situations in speech. In fact, "precious" is the addressee of Gollum's language: it is both himself and something else which at least superficially seems to be the Ring.

Aquí vemos que el famoso tesoro de Gollum se denomina originalmente precious. En una entrada de El Trujamán (2005: 25 enero), Julia y Manuel Sevilla Muñoz elogian esta solución, originaria de la traducción de los libros. Opinan que "en ambos casos se manifiesta el valor emocional que algo o alguien representa, utilizando una expresión que, al mismo tiempo, puede emplearse para hacer una valoración estrictamente material".

El poder del Anillo (y, por extensión, Sauron) sobre Sméagol es tal que llega a manifestarse en su habla mediante sus sonidos guturales. Estos sonidos, transcritos como gollum, rebautizan al personaje (Nagy 2006). Como explica Alsina (2012: 149), el uso de los nombres propios como elemento caracterizador es un recurso con una larga tradición en la narrativa inglesa. Curiosamente, de las dos identidades, la de Gollum es la única que emite ese sonido, pero nunca para referirse a sí mismo.

\subsection{Análisis del idiolecto de Gollum y de su traducción para el doblaje}

\subsubsection{Expresiones y léxico característicos}

Los rasgos idiosincrásicos léxicos son fácilmente reconocibles, y son los más representativos de cualquier idiolecto. Una elección desafortunada afectaría a la caracterización y a la integridad del personaje. Como puede apreciarse en el ejemplo 1, Gollum utiliza una adjetivación peculiar. Añadiendo el sufijo -able al verbo onomatopéyico crunch crea un neologismo especialmente gráfico. Esto se mantiene en la traducción, aunque con un adjetivo más convencional.

Ejemplo 1. Adjetivación dura y peculiar

\begin{tabular}{|l|l|}
\hline \multicolumn{1}{|c|}{ ORIGINAL } & \multicolumn{1}{c|}{ TRADUCCIÓN } \\
\hline $\begin{array}{l}\text { No, no birdses to eat. No crunchable } \\
\left.\text { birdses. (L2T, }{ }^{4} \text { min } 43\right)\end{array}$ & $\begin{array}{l}\text { No, no pájaros que comer. No crujientes } \\
\text { pajaritos. }\end{array}$ \\
\hline We must starve! (L2T, min 44) & ¡Moriremos de inanición! \\
\hline
\end{tabular}

En el polo opuesto, el uso de un término elevado como inanición disuena con la identidad del personaje, y crea una sensación de contradicción en el público, que Zabalbeascoa (2008) denomina "desubicación".

4. $\mathrm{L} 2 \mathrm{~T}=$ Las dos torres. $\mathrm{ERR}=$ El retorno del rey. 
Como comentábamos en el apartado 3.3, es importante que, una vez elegida la traducción para una determinada palabra, se mantenga a lo largo del texto. Es una cuestión de coherencia: Gollum tiene un vocabulario limitado y utiliza las mismas palabras, no tendría sentido traducirlas de forma diferente cada vez. Al hacerlo, alteraríamos injustificadamente su competencia lingüística y eliminaríamos su repetición propia (cf. apartado 5.2.4).

Ejemplo 2. Vocabulario limitado

\begin{tabular}{|l|l|}
\hline \multicolumn{1}{|c|}{ ORIGINAL } & \multicolumn{1}{c|}{ TRADUCCIÓN } \\
\hline $\begin{array}{l}\text { Sneaky little Hobbitses. Wicked. Tricksy. } \\
\text { False. (L2T, min 95) }\end{array}$ & $\begin{array}{l}\text { Sucios rastreros hóbbits. Malos, traidores, } \\
\text { falsos. }\end{array}$ \\
\hline $\begin{array}{l}\text { Wicked Men. Servants of Sauron. (L2T, } \\
\text { min 100) }\end{array}$ & Hombres malos. Siervos de Sauron. \\
\hline $\begin{array}{l}\text { Master betrayed us. Wicked, tricksy, } \\
\text { false. (L2T, min 141) }\end{array}$ & $\begin{array}{l}\text { Amo nos traicionó. Perverso, traidor, } \\
\text { falso. }\end{array}$ \\
\hline $\begin{array}{l}\text { I told you he was tricksy. I told you he } \\
\text { was false. (L2T, min 141) }\end{array}$ & $\begin{array}{l}\text { Te dije que era tramposo. Te dije que era } \\
\text { falso. }\end{array}$ \\
\hline Master is our friend. (L2T, min 141) & Amo es nuestro amigo. \\
\hline
\end{tabular}

Las equivalencias de master y false se mantienen a lo largo de la película, pero no los adjetivos wicked y tricksy, traducidos de dos formas diferentes, rompiendo con "la exigencia de recurrencia implícita en las formas idiolectales" (García de Toro 2009: 143) y diluyendo el idiolecto de Gollum. En esta escena, el personaje está de espaldas a la cámara, ninguna de las sincronías propias del doblaje (cf. apartado 2) explicaría esta variación.

No obstante, en casos como el del ejemplo 3 el cambio de solución podría deberse a razones de ajuste. Pese a que nice ya se había traducido por bueno con anterioridad, su aparición en una escena en la que Gollum habla en primer plano debió de obligar al ajustador a buscar una alternativa que no contuviera el fonema bilabial /b/. La articulación de este sonido, que no está presente en el original, podría haber provocado una asincronía labial demasiado evidente para el espectador, así que se opta por la palabra rico, que cuadra mejor con la imagen y ya existía en el idiolecto de Gollum. Esto hace que el cambio en el TM sea inapreciable y solo afecte ligeramente a la frecuencia de aparición de ambos términos. 
Ejemplo 3. La traducción de nice

\begin{tabular}{|l|l|}
\hline \multicolumn{1}{|c|}{ Original } & \multicolumn{1}{|c|}{ Traducción } \\
\hline $\begin{array}{l}\text { We be nice to them if they be nice to us. } \\
\text { (L2T, min 12) }\end{array}$ & $\begin{array}{l}\text { Seremos buenos con ellos si ellos buenos } \\
\text { con nosotros. }\end{array}$ \\
\hline $\begin{array}{l}\text { What does it eats? Is it tasty? (L2T, min } \\
43)\end{array}$ & ¿Qué come? ¿Está rico? \\
\hline $\begin{array}{l}\text { No. Not very nice at all, my love. (ERR, } \\
\text { min 25) }\end{array}$ & $\begin{array}{l}\text { No. No están muy ricos, desde luego, mi } \\
\text { amor. }\end{array}$ \\
\hline
\end{tabular}

El traductor puede encontrarse con el problema añadido de que la equivalencia elegida para un determinado término idiolectal no funcione en todos los contextos de uso del original. Es lo que ocurre en el ejemplo 4 con my love:

Ejemplo 4. La traducción de my love

\begin{tabular}{|l|l|}
\hline \multicolumn{1}{|c|}{ ORIGINAL } & \multicolumn{1}{c|}{ TRADUCCIÓN } \\
\hline $\begin{array}{l}\text { What's it saying, my precious, my love? Is } \\
\text { Sméagol losing his nerve? (ERR, min 24) }\end{array}$ & $\begin{array}{l}\text { ¿Qué insinúas, mi tesoro, mi amor? } \\
\text { ¿Sméagol pierde los nervios? }\end{array}$ \\
\hline Give us that, Déagol, my love. (ERR, min 3) & Danos eso, Déagol, querido. \\
\hline
\end{tabular}

My love, traducido por mi amor, es el vocativo que utiliza Gollum a veces para dirigirse a sí mismo (es decir, a Sméagol). Sin embargo, Sméagol también llama my love a su amigo Déagol en otra escena, en la que el traductor ha optado por una solución diferente. Como alternativa a my love, Gollum también recurre a la palabra precious, que, como explicamos en el apartado 5.1, también desempeña una función autorreferencial, además de designar al Anillo. A este uso corresponden los dos fragmentos siguientes, que sugieren que no se percibió este matiz:

Ejemplo 5. La traducción de precious

\begin{tabular}{|l|l|}
\hline \multicolumn{1}{|c|}{ ORIGINAL } & \multicolumn{1}{|c|}{ TRADUCCIÓN } \\
\hline $\begin{array}{l}\text { Yes, precious. False. They will cheat you, } \\
\text { hurt you, lie! (L2T, min 96) }\end{array}$ & $\begin{array}{l}\text { Sí, el tesoro. Falsos. Te engañan, te } \\
\text { sacuden, mienten. }\end{array}$ \\
\hline Yes, precious, she could. (L2T, min 103) & Sí, mi tesoro. Ella puede. \\
\hline
\end{tabular}

\subsubsection{Formas de autorreferencia}

La prueba más directa del trastorno de personalidad que padece Gollum son las múltiples formas de autorreferencia que utiliza a lo largo de las películas. Como comenta Arrizabalaga (2007: 268): 
Cuando la criatura se dirige a sí misma en los monólogos, la convivencia de Sméagol (el ser original) y Gollum (producto de la degradación ambiciosa del Anillo) se revela en una oscilación conflictiva entre 'we', que incluye a ambos, e 'it', la forma pronominal del tercero aludido.

El momento en que Frodo se refiere a Gollum como Sméagol al principio de Las dos torres supone un punto de inflexión en la trama, que se refleja en el hecho de que este retome el uso esporádico de la primera persona para referirse a sí mismo (Nagy 2006). Esta multiplicidad de identidades se aprecia en los extractos del siguiente ejemplo:

Ejemplo 6. La multiplicidad de identidades de Gollum

\begin{tabular}{|l|l|l|}
\hline PERSONAJE & \multicolumn{1}{|c|}{ ORIGINAL } & \multicolumn{1}{|c|}{ TRADUCCIÓN } \\
\hline Gollum & $\begin{array}{l}\text { Where would you be without } \\
\text { me? Gollum, gollum. I saved us. It } \\
\text { was me. We survived because of } \\
\text { me. (L2T, min 96) }\end{array}$ & $\begin{array}{l}\text { ¿Dónde estarias sin mí? Gollum, } \\
\text { gollum. Yo nos salvé. Nos salvé. } \\
\text { Sobrevivimos por mí. }\end{array}$ \\
\hline Gollum & $\begin{array}{l}\text { Sméagol. Why does it cry, } \\
\text { Sméagol? (L2T, min 141) }\end{array}$ & $\begin{array}{l}\text { Sméagol. ¿Por qué llora, } \\
\text { Sméagol? }\end{array}$ \\
\hline $\begin{array}{l}\text { Gollum } \\
\text { (Sméagol) }\end{array}$ & $\begin{array}{l}\text { We told him to go away. And } \\
\text { away he goes, precious. Gone, } \\
\text { gone, gone! Sméagol is free! } \\
\text { (L2T, min 97) }\end{array}$ & $\begin{array}{l}\text { Le dijimos que se fuera . Y él } \\
\text { se va, oh, tesoro. ¡Ido, ido, ido! } \\
\text { ¡Sméagol es libre! }\end{array}$ \\
\hline $\begin{array}{l}\text { Gollum } \\
\text { (Sméagol) }\end{array}$ & $\begin{array}{l}\text { What's it saying, my precious, my } \\
\text { love? (ERR, min 24) }\end{array}$ & $\begin{array}{l}\text { ¿Qué insinúas, mi tesoro, mi_ } \\
\text { amor? }\end{array}$ \\
\hline
\end{tabular}

En el primer extracto, Gollum trata a Sméagol de tú y le dice que ambos sobrevivieron gracias a él (Gollum). Lo paradójico de la situación es que yo, tú y nosotros son el mismo ser, pero la trama requiere esta distinción para reflejar la identidad fragmentada del personaje. Más adelante, Gollum también se dirige a Sméagol, aunque esta vez con el pronombre personal neutro de tercera persona, que en inglés se utiliza para animales y cosas. En el tercer fragmento, Sméagol se alegra de que ellos (solo Sméagol, aunque utilice el plural) le hayan dicho a él (Gollum) que se fuera, y acaba hablando de sí mismo en tercera persona (;Sméagol es libre!). Este caso es especialmente desconcertante, ya que demuestra que el desorden de Gollum no se limita a la confusión dicotómica Gollum/ Sméagol, sino que llega hasta el punto de no saber distinguir los referentes que hay tras la pronominalización. De todos modos, este rasgo idiolectal puede trasladarse al español de forma literal y, en general, se mantiene a lo largo de la historia, menos en los casos en los que se utiliza it, en cuya traducción no siempre se ha recurrido a la tercera persona (p. ej., insinúas). 
También es importante precisar que gollum (en minúscula) no constituye una forma de autorreferencia, puesto que es el sonido gutural que produce el personaje instintivamente y carece de intención comunicativa (cf. apartado 5.1).

\subsubsection{Deixis}

Gollum muestra cierta tendencia a la ambigüedad y a hablar de forma vaga y poco inteligible, algo que refleja su oscura personalidad. Gran parte de ese efecto se logra mediante el abuso de las referencias exofóricas o deixis en su discurso. En este apartado analizamos, básicamente, dos aspectos: los deícticos idiosincrásicos más recurrentes, en general, y los deícticos personales que utiliza para referirse a los hóbbits que lo acompañan (cf. apartado 4.3), en particular. Veamos el siguiente ejemplo:

Ejemplo 7. La traducción de los deícticos idiosincrásicos

\begin{tabular}{|l|l|}
\hline \multicolumn{1}{|c|}{ ORIGINAL } & \multicolumn{1}{c|}{ TRADUCCIÓN } \\
\hline $\begin{array}{l}\text { No! We won’t go back. Not there. Not to } \\
\text { him. (L2T, min 14) }\end{array}$ & ¡No! No volveremos. No allí. No a él. \\
\hline $\begin{array}{l}\text { No! No, master! They catch you! They } \\
\text { catch you! Don’t take it to him. He wants } \\
\text { the precious. Always he is looking for it. } \\
\text { (L2T, min 95) }\end{array}$ & $\begin{array}{l}\text { ¡No! ¡Amo, no! ¡Le cogerán! ¡Le cogerán! } \\
\text { Siempre lle está buscando. Él busca mi tesoro. }\end{array}$ \\
\hline $\begin{array}{l}\text { She's always hungry. She always needs to } \\
\text { feed. (ERR, min 25) }\end{array}$ & $\begin{array}{l}\text { Ella siempre está ansiosa. Siempre } \\
\text { necesita presas. }\end{array}$ \\
\hline
\end{tabular}

Curiosamente, pese a que los pronombres de tercera persona he, she o they suelen hacer de proformas (tienen un antecedente presente en el cotexto), aquí su valor es deíctico, porque remiten a la realidad extralingüística. Se trata, no obstante, de deixis distintas. Mientras que they remite al contexto inmediato, los protagonistas están en un lugar plagado de enemigos, he/him y she desempeñan una función referencial, y su referente es siempre el mismo: Sauron, el principal antagonista de la historia, y Ella-Laraña (Shelob, en la versión original), la araña gigante a la que Gollum admira y teme a partes iguales. Este fenómeno, fruto de la estrecha relación entre Gollum y los dos personajes, es constante a lo largo de la Trilogía, y ambos referentes pueden identificarse de forma fácil e inequívoca gracias al conocimiento que el espectador adquiere sobre la Tierra Media a medida que avanza la historia. Lo mismo ocurre con el deíctico espacial there, que en el ejemplo anterior equivale a Mordor, la región habitada por Sauron y sus tropas. El caso de it es algo diferente, puesto que 
a veces se utiliza como deíctico del Anillo, sin que este se haya mencionado previamente, y otras como proforma pronominal anafórica o catafórica, como en sus dos apariciones en el ejemplo anterior.

De nuevo, este tipo de referencias no entraña gran problema a nivel traductológico, ya que se pueden mantener tal cual en el TM. La que sí podría suponer un problema es la forma que tiene Gollum de dirigirse a los hóbbits. Observemos los siguientes fragmentos:

Ejemplo 8. La traducción de los deícticos personales para referirse a Frodo

\begin{tabular}{|l|l|}
\hline \multicolumn{1}{|c|}{ ORIGINAL } & \multicolumn{1}{c|}{ TRADUCCIÓN } \\
\hline $\begin{array}{l}\text { No! No, master! They catch you! They } \\
\text { catch you! Don't take it to him. (L2T, min } \\
\text { 95) }\end{array}$ & $\begin{array}{l}\text { ¡No! ¡Amo, no! ¡Le cogerán! iLe cogerán! } \\
\text { No se lo lleve a él. }\end{array}$ \\
\hline What does it eats? (L2T, min 44) & ¿Qué comẹ? \\
\hline $\begin{array}{l}\text { Master should be resting. Master needs to } \\
\text { keep up his strength. (L2T, min 48) }\end{array}$ & $\begin{array}{l}\text { Amo debería descansar. Amo necesita } \\
\text { recuperar fuerzas. }\end{array}$ \\
\hline $\begin{array}{l}\text { Look. Look. See what Sméagol finds? [...] } \\
\text { Eat them. Eat them! (L2T, min 97) }\end{array}$ & $\begin{array}{l}\text { Mire. Mire. ¿Ve lo que Sméagol } \\
\text { encuentra? [...] Comedlos. ¡Comedlos! }\end{array}$ \\
\hline
\end{tabular}

Gollum se dirige a Frodo utilizando tanto la segunda persona (p. ej., They catch you!) como la tercera (p. ej., What does it eats? y Master needs...). Como Frodo es el amo del Anillo e inspira cierto respeto en Gollum, el traductor traduce you sistemáticamente como usted y se mantiene coherente con su propuesta y no lo tutea en ningún momento. Pero esto tiene un efecto secundario en la traducción por el cambio de tenor interpersonal (Gregory \& Carrol 1978) que se hace en el original: cuando Gollum trata a Frodo de it, en español el verbo se conjuga igual que si lo estuviera tratando de usted y, si pensamos que el sujeto se elide en la mayoría de ocasiones, la distinción entre ambos es imperceptible (p. ej., ¿Qué come [usted/él]?). Quizás por ese motivo el traductor, para compensar, haya traducido los imperativos alternando el trato de usted con el de vos, como se aprecia en el último fragmento.

A quien sí tutea Gollum en la traducción, en cambio, es a Sam. El traductor debió de considerar necesario evidenciar la diferencia de trato entre los hóbbits, precisamente para demostrar que Gollum no profesa por Sam el mismo respeto que por el amo: 
Ejemplo 9. La traducción de los deícticos personales para referirse a Sam

\begin{tabular}{|l|l|}
\hline \multicolumn{1}{|c|}{ ORIGINAL } & \multicolumn{1}{c|}{ TRADUCCIÓN } \\
\hline $\begin{array}{l}\text { Give it to us raw and wriggling. You keep } \\
\text { nasty chips. (L2T, min 99) }\end{array}$ & $\begin{array}{l}\text { Dádnoslo vivo y coleando. Quédate con } \\
\text { tus repugnantes papas. }\end{array}$ \\
\hline
\end{tabular}

En cuanto a las formas de referirse a los dos hóbbits al mismo tiempo (ejemplo 10), Gollum utiliza tanto la segunda como la tercera persona, aunque, a diferencia de cuando se dirige exclusivamente a Frodo, en plural sí combina la forma de respeto (ustedes) con la de uso habitual (vosotros). De algún modo, esta alternancia compensa la neutralización de la distinción entre segunda y tercera persona en múltiples ocasiones, como ocurre con cumplir su voluntad:

Ejemplo 10. La traducción de los deícticos personales para referirse a los hóbbits

\begin{tabular}{|l|l|}
\hline \multicolumn{1}{|c|}{ ORIGINAL } & \multicolumn{1}{c|}{ TRADUCCIÓN } \\
\hline $\begin{array}{l}\text { We be nice to them if they be nice to us. } \\
\text { [...] We swears to do what you wants. } \\
\text { (L2T, min 12) }\end{array}$ & $\begin{array}{l}\text { Seremos buenos con ellos si ellos buenos } \\
\text { con nosotros. [...] Juramos cumplir su } \\
\text { voluntad. }\end{array}$ \\
\hline $\begin{array}{l}\text { This way, Hobbits. Follow me! (L2T, min } \\
\text { 14) }\end{array}$ & Por aquí, hóbbits. ¡Seguidme! \\
\hline Come, Hobbits! Come! (L2T, min 43) & ¡Vengan, hóbbits! ¡Vengan! \\
\hline
\end{tabular}

\subsubsection{Repetición}

"Repetitiousness, the automatism of language, reflects Gollum's deterioration into a state of control by corporeal drives and conditioned reflexes." Esta reflexión de Nagy (2006: 60) convierte la repetición en un elemento clave de la caracterización del personaje. El idiolecto de Gollum está marcado, fundamentalmente, por cuatro fenómenos reiterativos que hemos denominado reduplicación, reafirmación y renegación, polisíndeton y narración.

Ejemplo 11. La traducción de la reduplicación

\begin{tabular}{|l|l|}
\hline \multicolumn{1}{|c|}{ ORIGINAL } & \multicolumn{1}{c|}{ TRADUCCIÓN } \\
\hline $\begin{array}{l}\text { We swears to do what you wants. We } \\
\text { swears. (L2T, min 12) }\end{array}$ & $\begin{array}{l}\text { Juramos cumplir su voluntad. Juramos, } \\
\text { sí... }\end{array}$ \\
\hline Too risky. Too risky. (ERR, min 23) & Mucho riesgo. Demasiado. \\
\hline
\end{tabular}

La reduplicación está presente en casi todas las intervenciones de Gollum y consiste en la repetición total o parcial de lo que acaba de decir. Generalmente, 
las reduplicaciones se mantienen en el TM, aunque en ocasiones el traductor las sustituye por coloquialismos, elementos cómicos o marcas de oralidad. Otras veces, como en el segundo fragmento, la reduplicación debe sacrificarse para compensar la expansión del texto en la traducción y mantener la necesaria isocronía (cf. apartado 2).

Ejemplo 12. La traducción de la reafirmación y la renegación

\begin{tabular}{|c|c|}
\hline ORIGINAL & TRADUCCIÓN \\
\hline $\begin{array}{l}\text { We must go, yes. We must go at once. } \\
(\text { ERR, min } 7)\end{array}$ & Debemos ir, sí. Hay que seguir camino. \\
\hline $\begin{array}{l}\text { It's ours, it is, and we wants it! (L2T, min } \\
10)\end{array}$ & 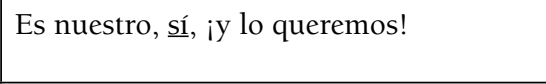 \\
\hline $\begin{array}{l}\text { No! We won't go back. Not there. Not to } \\
\text { him. They can't make us. (L2T, min 14) }\end{array}$ & $\begin{array}{l}\text { ¡No! No volveremos. No allí. No a él. No } \\
\text { pueden obligarnos. }\end{array}$ \\
\hline
\end{tabular}

La reafirmación y la renegación también abundan en el discurso de Gollum y se producen cuando el personaje afirma o niega el mismo enunciado más de una vez. Distinguimos dos clases de reafirmación: 1) la que se realiza con el adverbio yes al final de una oración afirmativa y 2) la que se forma repitiendo el sujeto y el verbo como autorrespuesta. Algo similar ocurre con la renegación, que puede producirse por no, not y sus diferentes formas. Tanto unas como otras se han traducido por los adverbios sí y no, respectivamente.

Ejemplo 13. La traducción del polisíndeton

\begin{tabular}{|c|c|}
\hline ORIGINAL & TRADUCCIÓN \\
\hline $\begin{array}{l}\text { No. Ashes and dust and thirst there is, and } \\
\text { pits, pits, pits. And Orcses, thousands } \\
\text { of Orcses. And always the Great Eye } \\
\text { watching, watching. (L2T, min 14) }\end{array}$ & $\begin{array}{l}\text { No. Ceniza } y \text { polvo } y \text { sed solo hay, } y \text { fosos, } \\
\text { fosos, fosos. } \underline{Y} \text { orcos, orcos a millares. } \underline{Y} \\
\text { siempre Gran Ojo vigilante, vigilante. }\end{array}$ \\
\hline $\begin{array}{l}\text { All dead. All rotten. Elves and Men and } \\
\text { Orcses. (L2T, min 45) }\end{array}$ & $\begin{array}{l}\text { Todos muertos. Todos putrefactos. Elfos, } \\
\text { hombres } ¥ \text { orcos. }\end{array}$ \\
\hline
\end{tabular}

El polisíndeton es una figura retórica que consiste en la repetición de las conjunciones. Su presencia es importante para el TM, porque refuerza la impresión de que Gollum habla sin planificar su discurso. A pesar de ello, no siempre se mantiene este rasgo en la traducción.

Por otro lado, observamos que, en algunas situaciones, Gollum tiende a explicar lo que acaba de ocurrir, a modo de narrador externo que describe la acción. En este caso, se trata de un fenómeno supraoracional que, prácticamente, se puede reproducir en la traducción palabra por palabra. 
Ejemplo 14. La traducción del fenómeno supraoracional

\begin{tabular}{|l|l|}
\hline \multicolumn{1}{|c|}{ ORIGINAL } & \multicolumn{1}{c|}{ TRADUCCIÓN } \\
\hline $\begin{array}{l}\text { To the Gate, to the Gate! To the Gate, the } \\
\text { master says. Yes! (L2T, min 14) }\end{array}$ & $\begin{array}{l}\text { ¡Hasta la Puerta! La Puerta, ordena el } \\
\text { amo, } \underline{\text { sí. }}\end{array}$ \\
\hline $\begin{array}{l}\text { Master says to show him the way into } \\
\begin{array}{l}\text { Mordor. So good Sméagol does, Master } \\
\text { says so. (L2T, min 62) }\end{array}\end{array}$ & $\begin{array}{l}\text { Amo pide que le muestre el camino a } \\
\text { Mordor y el buen Sméagol así hace, amo } \\
\text { lo dijo. }\end{array}$ \\
\hline
\end{tabular}

\subsubsection{Anomalías sintácticas}

En el idiolecto de Gollum abundan las construcciones anómalas. De todas las que hemos observado, las más representativas son el hipérbato y las omisiones, ejemplificadas a continuación:

Ejemplo 15. La traducción del hipérbato

\begin{tabular}{|l|l|}
\hline \multicolumn{1}{|c|}{ ORIGINAL } & \multicolumn{1}{|c|}{ TRADUCCIÓN } \\
\hline $\begin{array}{l}\text { And we are famished! Yes! Famished we } \\
\text { are, precious! (L2T, min 43) }\end{array}$ & $\begin{array}{l}\text { ¡Estamos hambrientos, sí! ¡Hambrientos } \\
\text { estamos, oh, tesoro! }\end{array}$ \\
\hline $\begin{array}{l}\text { Dead? No, you cannot kill them. (L2T, } \\
\text { min 49) }\end{array}$ & ¿Muertos? No, matarlos no puedes. \\
\hline
\end{tabular}

En el TM se ha tendido a mantener los hipérbatos donde el personaje los utiliza en inglés, incluso se han introducido algunos que no aparecían en el TO, que compensan su pérdida en otros puntos del texto. En la traducción se utilizan de tres clases de omisiones: del verbo, del argumento verbal y del artículo. Las dos primeras corresponden ocasionalmente a omisiones del original, pero se utilizan sobre todo como estrategia de compensación (cf. apartado 5.2.6). La tercera forma de omisión, en cambio, es exclusiva de la traducción, y su uso esporádico contribuye a resaltar las carencias lingüísticas del personaje:

Ejemplo 16. La traducción de las omisiones

\begin{tabular}{|l|l|}
\hline \multicolumn{1}{|c|}{ ORIGINAL } & \multicolumn{1}{c|}{ TRADUCCIÓN } \\
\hline Orcs don't know it. (L2T, min 43) & $\varnothing$ Orcos no $\varnothing$ conocen. \\
\hline $\begin{array}{l}\text { No. Ashes and dust and thirst there is } \\
\begin{array}{l}\text { l...]. And always the Great Eye watching, } \\
\text { watching. (L2T, min 14) }\end{array}\end{array}$ & $\begin{array}{l}\text { Ceniza y polvo y sed solo hay [...]. Y } \\
\text { siempre } \varnothing \text { Gran Ojo vigilante, vigilante. }\end{array}$ \\
\hline
\end{tabular}




\subsubsection{Anomalías en las formas verbales}

El paradigma verbal de Gollum presenta, a grandes rasgos, cuatro anomalías: 1) la falta de concordancia entre sujeto y verbo, 2) la construcción incorrecta de las oraciones condicionales, 3) la omisión ocasional del verbo auxiliar en algunos tiempos compuestos y 4) el uso de tiempos verbales perfectamente gramaticales, pero contextualmente incorrectos.

La no concordancia entre sujeto y verbo es muy habitual en el idiolecto de Gollum. A veces, refleja la confusión de sujetos, porque tiene diversas formas de referirse a sí mismo (cf. apartado 5.2.2), pero casi siempre se debe a que aplica irregularmente la forma de presente de la tercera persona del singular. Esta peculiaridad morfosintáctica tiene un origen fonético: la predilección de Gollum por el fonema sibilante /s/ (cf. apartado 5.2.7). Su distribución en el texto es desigual y su traducción, especialmente compleja.

Las formas verbales en español son más largas que en inglés, por lo que se ha optado por elidir el sujeto en la mayoría de los casos para reducir la extensión de la traducción. Aunque en inglés la elipsis del sujeto es agramatical, es la práctica habitual en español. Como resultado de esta elisión, el sujeto solo se manifiesta en la oración por medio de la conjugación del verbo. Si se utilizara otra forma verbal para intentar reproducir el problema de concordancia entre sujeto y verbo presente en el original, no se indicaría el sujeto de la oración. Esto limita las posibilidades del traductor, que utiliza otros recursos como la omisión para reflejar la irregularidad verbal característica del habla de Gollum.

Ejemplo 17. La traducción de la falta de concordancia entre sujeto y verbo

\begin{tabular}{|l|l|}
\hline \multicolumn{1}{|c|}{ ORIGINAL } & \multicolumn{1}{c|}{ TRADUCCIÓN } \\
\hline Sméagol look after master. (ERR, min 75) & Sméagol cuida del amo. \\
\hline $\begin{array}{l}\text { We swears to do what you wants. We } \\
\text { swears. (L2T, min 12) }\end{array}$ & $\begin{array}{l}\text { Juramos cumplir su voluntad. } \varnothing \text { Juramos, } \\
\text { sí... }\end{array}$ \\
\hline $\begin{array}{l}\text { But they knows. They knows. They } \\
\text { suspects us. (ERR, min 24) }\end{array}$ & $\begin{array}{l}\text { Pero ellos } \varnothing \text { saben. Lo saben. Sospechan } \\
\text { de nosotros. }\end{array}$ \\
\hline
\end{tabular}

Como puede apreciarse, el traductor conjuga correctamente todos los verbos. En nuestra opinión, no hay ninguna razón para no conservar el error de concordancia en las oraciones en las que, por razones de sincronía o estilo, sí se ha decidido mantener el sujeto, como pasa con Pero ellos saben. Además, podría priorizarse la forma sobre el significado y utilizar la segunda persona del singular, sabes, que también acaba en $s$. En su lugar, se ha recurrido a dos tipos de omisión (cf. apartado 5.2.5) para recrear la agramaticalidad en el TM: 
la omisión del verbo, directamente, o la de algún argumento verbal (p. ej., el complemento directo en Pero ellos saben).

Siguiendo con la segunda anomalía, en el ejemplo 18 vemos que Gollum es incapaz de construir una oración condicional. Se trata de un claro caso de primer condicional, que formulado correctamente sería: We'll be nice to them if they are nice to us. Pero él se limita a usar la conjunción condicional y elimina las diferencias verbales entre condición y resultado utilizando en ambos casos el infinitivo. La omisión verbal en la segunda parte de la traducción trata de evocar este efecto, aunque podría complementarse con el uso de la forma de infinitivo, en lugar de la de futuro.

Ejemplo 18. La traducción de la omisión ocasional del verbo auxiliar en tiempos compuestos

\begin{tabular}{|c|c|}
\hline ORIGINAL & TRADUCCIÓN \\
\hline $\begin{array}{l}\text { We } \varnothing \text { be nice to them if they be nice to } \\
\text { us. (L2T, min } 12 \text { ) }\end{array}$ & $\begin{array}{l}\text { Seremos buenos con ellos si ellos } \underline{\varnothing} \\
\text { buenos con nosotros. }\end{array}$ \\
\hline
\end{tabular}

El tercer fenómeno verbal se ha eliminado casi por completo en la traducción. Es cierto que, si la forma verbal utilizada en español carece de verbo auxiliar (como ocurre con el futuro simple, por ejemplo), el efecto no puede reproducirse igual que en el original, pero el traductor podría haberlo recreado con algún otro recurso; por ejemplo, con el presente en el primer fragmento, que evitaría alterar el sujeto: Y después cogemos el tesoro iy somos el amo!

Ejemplo 19. La traducción de tiempos verbales gramaticales pero contextualmente incorrectos

\begin{tabular}{|l|l|}
\hline \multicolumn{1}{|c|}{ ORIGINAL } & \multicolumn{1}{c|}{ TRADUCCIÓN } \\
\hline $\begin{array}{l}\text { And then we } \varnothing \text { take the precious and we } \\
\varnothing \text { be the master! (L2T, min 102) }\end{array}$ & $\begin{array}{l}\text { Y después cogeremos el tesoro iy seremos } \\
\text { el amo! }\end{array}$ \\
\hline $\begin{array}{l}\text { But the fat Hobbit, he knows. } \varnothing \text { Eyes } \varnothing \\
\text { always watching. (L2T, min 102) }\end{array}$ & $\begin{array}{l}\text { Pero el hóbbit seboso lo sabe. } \varnothing \text { Ojos } \varnothing \\
\text { siempre mirando. }\end{array}$ \\
\hline $\begin{array}{l}\text { He took it! He took it. I } \varnothing \text { seen him. } \\
(\text { ERR, min 82) }\end{array}$ & ¡Ha sido él! ¡Él lo cogió! Yo lo vi. \\
\hline
\end{tabular}

Lo mismo podría hacerse con los tiempos verbales mal usados, cuyas traducciones al español vuelven a pecar de corrección. 
Ejemplo 20. La traducción de tiempos verbales mal empleados

\begin{tabular}{|l|l|}
\hline \multicolumn{1}{|c|}{ ORIGINAL } & \multicolumn{1}{|c|}{ TRADUCCIÓN } \\
\hline $\begin{array}{l}\text { No, master! They catch you! They catch } \\
\text { you! (L2T, min 95) }\end{array}$ & ¡Amo, no! ¡Le cogerán! ¡Le cogerán! \\
\hline Very lucky we find you. (L2T, min 42) & Suerte que os encontramos. \\
\hline
\end{tabular}

La última frase es algo curiosa, porque el verbo puede leerse como si estuviera conjugado en presente (como en el original) o en pretérito perfecto simple (el tiempo correcto), pero es improbable que el público perciba la ambigüedad en tiempo real.

Observamos que la traducción contiene un fenómeno verbal inexistente en el original: el uso alternado de imperativos e infinitivos. La introducción de esta agramaticalidad, habitual en español, nos parece un buen método de compensación, pero creemos que la opción más coherente para el personaje sería utilizar siempre una de las dos formas.

Ejemplo 21. La traducción de imperativos e infinitivos

\begin{tabular}{|l|l|}
\hline \multicolumn{1}{|c|}{ ORIGINAL } & \multicolumn{1}{|c|}{ TRADUCCIÓN } \\
\hline $\begin{array}{l}\text { Wake up! Wake up! Wake up, sleepies. } \\
\text { (ERR, min 7) }\end{array}$ & $\begin{array}{l}\text { ¡Despertad! ¡Despertad! Arriba, } \\
\text { dormilones. }\end{array}$ \\
\hline Eat them. Eat them! (L2T, min 97) & Comedlos. ¡Comedlos! \\
\hline Hide! Hide! Hide! (ERR, min 54) & ¡Esconder! ¡Esconder! ¡Esconder! \\
\hline
\end{tabular}

\subsubsection{Rasgos fonéticos}

En el idiolecto de Gollum, el marcador fonético por antonomasia es la prolongación recurrente del fonema sibilante/s/ o seseo. Nagy (2006: 59-60) explica el origen de este fenómeno:

The sibilance of his phonology derives from a sort of physical conditioning: the lack of articulation (because his language for a long time did not function as communication, being only monologue for which no clear articulation is necessary) and the need for whispered and concealed speech.

Este rasgo ya estaba presente en los libros de Tolkien, en los que se marcaba mediante la duplicación ocasional de la s, como en "We musstn't rissk our neck, musst we, precious?" (Tolkien 1966: 638). En el TM se ha conservado la prolongación de la sibilancia, aunque se sustituye con frecuencia por el fonema fricativo $/ \mathrm{J} /$, cambio que no podemos atribuir a una fase concreta del proceso de doblaje. El fonema plosivo /t/ también se pronuncia con especial énfasis en 
el original en palabras como it, stole o did, igual que ocurre con /p/ en precious. Estos rasgos, sin embargo, se han omitido en el TM o son inapreciables.

\subsubsection{Plurales anómalos}

Hay ocasiones en las que Gollum, para extender intuitivamente la sibilancia al final de palabras como birds, eyes, jackets, Hobbits y Orcs, "aplica una regla de plural en '-es' a términos que ya se encuentran en plural" (Arrizabalaga 2007: 267). Este recurso morfológico, que daría como resultado palabras como órcoses o hóbbitses, no se reproduce en la versión en español, en la que todos los plurales se forman siguiendo la normativa:

Ejemplo 24. La traducción de plurales anómalos

\begin{tabular}{|l|l|}
\hline \multicolumn{1}{|c|}{ ORIGINAL } & \multicolumn{1}{c|}{ TRADUCCIÓN } \\
\hline Hurry, Hobbitses. (L2T, min 42) & Prisa, hóbbits. \\
\hline $\begin{array}{l}\text { And Orcses, thousands of Orcses. (L2T, } \\
\text { min 14) }\end{array}$ & Y orcos, orcos a millares. \\
\hline $\begin{array}{l}\text { No, no birdses to eat. No crunchable } \\
\text { birdses. (L2T, min 43) }\end{array}$ & $\begin{array}{l}\text { No, no pájaros que comer. No crujientes } \\
\text { pajaritos. }\end{array}$ \\
\hline
\end{tabular}

En el último fragmento se observa el uso del diminutivo, quizás para compensar la pérdida del doble plural, pero no parece haber motivo para la omisión de la idiosincrasia, más allá de la tendencia ya observada por Goris (1993; cf. apartado 3.3).

\section{Conclusiones}

Si cada persona es un mundo, cada idiolecto es la expresión lingüística particular y exclusiva de ese mundo, y contribuye a identificar unívocamente a un personaje. Por eso, no es extraño que soluciones válidas para un individuo no sean aplicables a otros. La estrategia de traducción adoptada en cada caso dependerá de diversos factores, como la tipología textual, la relevancia del personaje, el papel del idiolecto en el texto, etc. Este razonamiento puede llevarnos a pensar que el análisis de las idiosincrasias de un personaje carece de interés para la investigación, pero lo cierto es que su interpretación es extrapolable a todo tipo de textos idiolectales. Lejos de aportar soluciones universales para la recreación de las idiosincrasias, lo que pretende este escrito es ofrecer al traductor una visión global del problema que le permita enfrentarse a ellas con conocimiento y tomar decisiones fundadas. 
La comparación minuciosa del original con la traducción nos confirma lo que ya observaron algunos de los especialistas mencionados en el apartado 3 (cf. Goris 1993; Sánchez Iglesias 2005): si bien el doblaje conserva parcialmente el idiolecto de Gollum y su funcionalidad, a menudo se normalizan las idiosincrasias injustificadamente. El seseo constante, por ejemplo, uno de los rasgos más representativos del personaje, se mantiene en el TM, igual que algunos fenómenos idiolectales de carácter sintáctico, pero las idiosincrasias morfológicas se neutralizan completamente. Esta carencia del TM se ha mitigado gracias al uso acertado de la compensación. A nivel léxico, el traductor ha hecho una selección consciente y meditada para reconstruir el vocabulario y el repertorio de expresiones de Gollum, pero podría haber evitado la alternancia de equivalencias para una sola forma del original.

Por último, nos gustaría destacar que las soluciones de traducción del doblaje podrían contrastarse con las de otras modalidades, como el subtitulado, o con la traducción de los libros de Tolkien. Eso ampliaría así el estudio sobre el tratamiento del idiolecto en el panorama traductológico. Pese a la parcialidad de los resultados, creemos que este artículo demuestra que la corrección no siempre conlleva adecuación. Deseamos concienciar a los traductores de la importancia de conocer y dominar las nociones teóricas fundamentales sobre el idiolecto y la variación lingüística en general y sus implicaciones para el tratamiento adecuado de los rasgos idiosincrásicos presentes, no solo en productos audiovisuales, sino en todo tipo de ficción. Al fin y al cabo, ¿qué sería de Gollum sin su tesoro?

\section{Referencias bibliográficas}

Alcaraz Varó, Enrique \& María Antonia Martínez Linares. (1997) Diccionario de lingüística moderna. Barcelona: Ariel.

AlsinA, Victòria. (2005) "Adaptaciones cinematográficas de obras literarias: el caso de Jane Austen." En: Zabalbeascoa, Patrick; Laura Santamaria \& Frederic Chaume (eds.) 2005. La traducción audiovisual: investigación, enseñanza y profesión. Granada: Comares, pp. 53-64.

Alsina, Victòria. (2012) "The translation of idiolect in children's literature. The Witches and Matilda by Roald Dahl." En: Fischer, Martin B. \& Maria Wirf (eds.) 2012. Translating Fictional Dialogue for Children and Young People (1 ${ }^{\mathrm{a}}$ ed). Berlín: Frank \& Timme, pp. 145-164.

ARRizABALAGA, María Inés. (2007) "Hobbits \& Riddles: reflexiones sobre la traducción al castellano de la obra de J. R. R. Tolkien." Lenguaje: Revista de la Escuela de Ciencias del Lenguaje de la Universidad del Valle 35:1, pp. 245-275. 
BAÑOS-PiÑERO, Rocío \& Frederic Chaume. (2009) "Prefabricated orality: A challenge in audiovisual translation." Intralinea: Online Translation Journal 6. Versión electrónica: <http://www.intralinea.org/specials/article/1714>

BARTOLL, Eduard. (2015) Introducción a la traducción audiovisual. Barcelona: Editorial UOC.

BILGRAMI, Akeel. (2005) Idiolect. En: Honderich, Ted (ed.) 2005. The Oxford Companion to Philosophy ( $2^{\mathrm{a}}$ ed.). Versión electrónica: <http://www. oxfordreference.com/view/10.1093/acref/9780199264797.001.0001/ acref-9780199264797-e-1177?rskey=MPyXr9\&result=1177\&q=>

CATFORD, John Cunnison. (1965) A Linguistic Theory of Translation. Londres: Oxford University Press.

ChAume, Frederic. (2001). "La pretendida oralidad de los textos audiovisuales y sus implicaciones en traducción." En: Agost, Rosa (ed.) 2001. La traducción en los medios audiovisuales. Castelló de la Plana: Publicacions de la Universitat Jaume I.

Chaume, Frederic. (2003) Doblatge i subtitulació per a la TV. Vic: Eumo.

Chaume, Frederic. (2012) Audiovisual translation: Dubbing. Kinderhook: St. Jerome.

Coleridge, Samuel Taylor y John T. SHAwCross. (1907) Biographia Literaria. Oxford: Clarendon Press.

CORRIUS, Montse \& Patrick Zabalbeascoa. (2011) "Language Variation in source texts and their translations: The case of L3 in film translation." Target: International Journal of Translation Studies 23:1, pp. 113-130.

COSTA, Walter Carlos. (2012) "Traducción literaria, variedad e idiolecto." Aletria: Revista de Estudios de Literatura 22:1, pp. 83-89.

COUlTHARD, Malcolm. (2004) "Author Identification, Idiolect, and Linguistic Uniqueness." Applied Linguistics 25:4, pp. 431-447.

ELdOBlajE.COM. (2017a) El señor de los anillos: Las dos torres. Versión electrónica: <http://www.eldoblaje.com/datos/FichaPelicula.asp?id=3446>

EldoblajE.COM. (2017b) El señor de los anillos: El retorno del rey. Versión electrónica: <http://www.eldoblaje.com/datos/FichaPelicula.asp?id=5080>

GARCíA DE TORO, Ana Cristina. (1994) "Idiolecto y traducción.” En: Bueno García, Antonio; Ramiro Valderrama \& Juan Miguel Zarandona (eds.) 1994. La traducción de lo inefable: Jorge Guillén, la emoción ontológica: Claude Simon, el diálogo de la voz interior: actas del I Congreso Internacional de Traducción e Interpretación de Soria. Soria: Diputación Provincial de Soria, pp. 91-101.

GARCÍA DE TORO, Ana Cristina. (2009) La traducción entre lenguas en contacto: catalán y español. Berna: Peter Lang.

GARCía LÓPEZ, Rosario. (2004) Guía didáctica de la traducción de textos idiolectales: texto literario y texto de opinión. Coruña: Netbiblo. 
GORIS, Olivier. (1993) "The Question of French Dubbing: Towards a Frame for Systematic Investigation." Target: International Journal of Translation Studies 5:2, pp. 169-190.

GREGORY, Michael \& Suzanne Carroll. (1978) Language and Situation: Language Varieties and their Social Contexts. Londres: Routledge $\&$ Kegan Paul.

Hatim, Basil \& Ian Mason. (1991) Discourse and the Translator (2. ${ }^{a}$ ed.). Londres: Longman.

HATIM, Basil \& Ian Mason. (1997) The Translator as Communicator. Londres: Routledge.

JACKSON, Peter (dir.) (2002). El señor de los anillos: Las dos torres [Película, versión extendida]. EE. UU./Nueva Zelanda: New Line Cinema.

JACKSON, Peter (dir.) (2003) El señor de los anillos: El retorno del rey [Película, versión extendida]. EE. UU. Y Nueva Zelanda: New Line Cinema.

LÓPEZ RÚA, Paula. (1997) "The Translation of Idiolects in The Catcher in the Rye: An Approach through Lexicalized Structures." Miscelánea: A Journal of English and American Studies 18, pp. 139-158.

MAYORAL, Roberto. (1999) "La traducción de la variación lingüística." Hermeneus: Revista de la Facultad de Traducción e Interpretación de Soria 1 (extra.), pp. 1-219.

NAGY, Gergely. (2006) "The 'Lost' Subject of Middle-earth: The Constitution of the Subject in the Figure of Gollum in The Lord of the Rings." Tolkien Studies: An Annual Scholarly Review 3, pp. 57-79.

Newmark, Peter. (1988) A Textbook of Translation. Hemel Hempstead: Prentice Hall International.

O’Donnell, William Robert \& Loreto Todd. (1980) Variety in Contemporary English. Londres: Allen \& Unwin.

RosA, Alexandra Assis. (2001) "Features of Oral and Written Communication in Subtitling." En: Gambier, Yves \& Henrik Gottlieb (eds.) 2001. (Multi)Media Translation. Ámsterdam \& Filadelfía: John Benjamins, pp. 213-222.

SÁNCHEZ IGLESIAS, Jorge Juan. (2001) "Restricciones semántico-textuales de la traducción del idiolecto: Lessico Famigliare de Natalia Ginzburg." En: Barr, Anne; Rosario Martín \& Jesús Torres (eds.) 2001. Últimas corrientes teóricas en los estudios de traducción y sus aplicaciones. Salamanca: Universidad de Salamanca, pp. 703-717.

SÁNCHEZ IGLESIAS, Jorge Juan. (2005) "El idiolecto y su traducción: tres ejemplos italianos." Revista de la Sociedad Española de Italianistas 3, pp. 165-184.

SEVILla Muñoz, Julia \& Manuel Sevilla Muñoz. (2005) "Un acierto de traducción conceptual." El Trujamán: Revista diaria de traducción. Versión electrónica: <http://cvc.cervantes.es/trujaman/anteriores/enero_05/25012005.htm>

THE TOLKIEN SOCIETY. (2017) J. R. R. Tolkien: A Biographical Sketch. Versión electrónica: <http://www.tolkiensociety.org/tolkien/biography.html> 
TOLKIEN, John Ronald Reuel. (1966) The Lord of the Rings (2. ${ }^{a}$ ed.). Londres: Book Club Associates.

TOLKIEN, John Ronald Reuel. (1975) "Guide to the Names in The Lord of the Rings.” En: Lobdell, Jared (ed.) 1975. A Tolkien Compass (1. ${ }^{a}$ ed.). Chicago: Open Court. Versión electrónica: <http://tolkien.ro/text/JRR\%20Tolkien\%20 -\%20Guide\%20to\%20the\%20Names\%20in\%20The\%20Lord\%20of\%20the\%20 Rings.pdf>

ZabalBEASCOA, Patrick. (2008) "La credibilidad de los diálogos traducidos para audiovisuales." En: Brumme, Jenny (ed.) 2008. La oralidad fingida: descripción y traducción: teatro, cómic y medios audiovisuales. Madrid \& Fráncfort del Meno: Iberoamericana \& Vervuert, pp. 155-176.

\section{Referencias filmográficas}

The Lord of the Rings: The Fellowship of the Ring (El señor de los anillos: La comunidad del anillo, Peter Jackson 2001)

The Lord of the Rings: The Two Towers (El señor de los anillos: Las dos torres, Peter Jackson 2002)

The Lord of the Rings: The Return of the King (El señor de los anillos: El retorno del rey, Peter Jackson 2003)

\section{NOTAS BIOGRÁFICAS / BIONOTES}

GUILLERMO PARRA LÓPEZ es traductor audiovisual y asistente de docencia en el Departamento de Traducción y Ciencias del Lenguaje de la Universidad Pompeu Fabra (UPF), en Barcelona. Allí imparte las asignaturas de Traducción y medios de comunicación y Fundamentos de la traducción, por la que recibió el Premio del Consejo Social a la Calidad en la Docencia en 2015. Guillermo tiene un Grado en Traducción e Interpretación y un Máster en Estudios de Traducción, y actualmente está realizando el doctorado sobre la traducción del lenguaje alterado por las drogas en el cine bajo la supervisión de Patrick Zabalbeascoa y Eduard Bartoll. Es autor de las publicaciones Disorderly speech and its translation: fear and loathing among letters (Parra López, 2016) y Translating drug-induced language variation in cinema (Parra López, en prensa).

DR. EDUARD BARTOLL es traductor audiovisual, sobre todo del inglés y el alemán al catalán y al español. Ha subtitulado más de 500 películas. También traduce obras de teatro, a menudo sobretituladas. Enseñó en la Universitat Pompeu Fabra de Barcelona, como lector en traducción del alemán y el inglés al catalán y al español. También ha trabajado en la Universitat Autònoma de Barcelona, Leipzig Universität, Heidelberg Universität, Universidade Federal do Ceará, etc. 
En 2012 publica un libro en catalán sobre la subtitulación y en 2015 publica otro en español sobre la traducción audiovisual. Ha escrito varios artículos sobre traducción audiovisual, sobre todo subtitulación, y ha asistido a numerosos congresos, tales como Media for All (1, 2, 3 y 6), Languages and the Media (5 y 6), etc.

GUILLERMO PARRA LÓPEZ is an audiovisual translator and teaching assistant in the Translation and Language Sciences Department of the Pompeu Fabra University (UPF), in Barcelona. He teaches Translation and the Media and Fundamentals of Translation, for which he received the UPF Social Council Award for Teaching Quality in 2015. Guillermo holds a Bachelor's Degree in Translation and Interpreting and a Master's Degree in Translation Studies and is currently doing a $\mathrm{PhD}$ on the translation of disorderly speech under the supervision of Patrick Zabalbeascoa and Eduard Bartoll. He is the author of Disorderly speech and its translation: fear and loathing among letters (Parra López, 2016) and Translating drug-induced language variation in cinema (Parra López, in press).

DR EDUARD BARTOLL is an audiovisual translator, mainly from English and German into Catalan and Spanish. He has subtitled more than 500 films and he translates theatre plays too, often for surtitles. He has worked at the university Pompeu Fabra, in Barcelona, where he was a lecturer in translation from German and English into Catalan and Spanish. He has also worked at the Universitat Autònoma de Barcelona, and at other universities, like Leipzig Universität, Heidelberg Universität, Universidade Federal do Ceará, etc.

In 2012 published a book in Catalan about Subtitling and in 2015 he published another book in Spanish about audiovisual translation. He has written several articles on audiovisual translation, especially on subtitling, and has attended many conferences, such as Media for All (1st, 2nd and 3rd), Languages and the Media (5th and 6th), etc. 


\section{AIMS / OBJETIVOS / OBJECTIUS}

MonTI (Monographs in Translation and Interpreting) is an academic, peer-reviewed and international journal fostered by the three public universities with a Translation Degree in the Spanish region of Valencia (Universitat d'Alacant, Universitat Jaume I de Castelló and Universitat de València).

Each issue will be thematic, providing an in-depth analysis of translationand interpreting-related matters that meets high standards of scientific rigour, fosters debate and promotes plurality. Therefore, this journal is addressed to researchers, lecturers and specialists in Translation Studies.

MonTI will publish one issue each year, first as a hard copy journal and later as an online journal.

In order to ensure both linguistic democracy and dissemination of the journal to the broadest readership possible, the hard-copy version will publish articles in German, Spanish, French, Catalan, Italian and English. The online version is able to accommodate multilingual versions of articles, and it will include translations into any other language the authors may propose and an attempt will be made to provide an English-language translation of all articles not submitted in this language.

Further information at:

http://dti.ua.es/es/monti-english/monti-contact.html

$* * * * *$

MonTI es una revista académica con vocación internacional promovida por las universidades públicas valencianas con docencia en traducción e interpretación (Universidad de Alicante, Universidad Jaume I de Castellón y Universidad de Valencia). 
Nuestra revista pretende ante todo centrarse en el análisis en profundidad de los asuntos relacionados con nuestra interdisciplina a través de monográficos caracterizados por el rigor científico, el debate y la pluralidad. Por consiguiente, la revista está dirigida a investigadores, docentes y especialistas en estudios de traducción.

MonTI publicará un número monográfico anual, primero en papel y a continuación en edición electrónica. Igualmente y con el fin de alcanzar un equilibrio entre la máxima pluralidad lingüística y su óptima difusión, la versión en papel admitirá artículos en alemán, castellano, catalán, francés, italiano o inglés, mientras que la edición en Internet aceptará traducciones a cualquier otro idioma adicional y tratará de ofrecer una versión en inglés de todos los artículos.

Más información en:

http://dti.ua.es/es/monti/monti.html

$$
* * * * *
$$

MonTI és una revista acadèmica amb vocació internacional promoguda per les universitats públiques valencianes amb docència en traducció i interpretació (Universitat d'Alacant, Universitat Jaume I de Castelló i Universitat de València).

La nostra revista pretén sobretot centrar-se en l'anàlisi en profunditat dels assumptes relacionats amb la nostra interdisciplina a través de monogràfics caracteritzats pel rigor científic, el debat i la pluralitat. Per tant, la revista va dirigida a investigadors, docents i especialistes en estudis de traducció.

MonTI publicarà un número monogràfic anual, primer en paper i a continuació en edició electrònica. Igualment, i a fi d'aconseguir un equilibri entre la màxima pluralitat lingüística i la seua difusió òptima, la versió en paper admetrà articles en alemany, castellà, català, francès, italià o anglès, mentre que l'edició en Internet acceptarà traduccions a qualsevol altre idioma addicional i tractarà d'oferir una versió en anglès de tots els articles.

Més informació a:

http://dti.ua.es/es/monti-catalan/monti-contacte.html 